\title{
MRI Findings in Acute Tonsillar Infections
}

\author{
(D). Heikkinen, (D). Nurminen, (D). Velhonoja, (D). Irjala, (D). Soukka, (D). Happonen,
} (D) M. Nyman, D. Mattila, and (D). Hirvonen

\section{ABSTRACT}

BACKGROUND AND PURPOSE: Previous literature is vague on the prevalence and exact nature of abscesses in tonsillar infections, ranging from intratonsillar and peritonsillar collections to deep extension involving the parapharyngeal and retropharyngeal spaces. MR imaging has excellent diagnostic accuracy in detecting neck infections and can potentially clarify this issue. We sought to characterize the spectrum of MR imaging findings regarding tonsillar infections.

MATERIALS AND METHODS: We conducted a retrospective cohort study of emergency neck MR imaging scans of patients with tonsillar infections. Imaging data were assessed in terms of signs of infection and the location of abscesses and were compared with clinical findings, final diagnoses, and surgical findings as reference standards.

RESULTS: The study included 132 patients with tonsillar infection. Of these, 110 patients (83\%) had $\geq 1$ abscess (99 unilateral, 11 bilateral; average volume, $3.2 \mathrm{~mL}$ ). Most abscesses were peritonsillar, and we found no evidence of intratonsillar abscess. Imaging showed evidence of parapharyngeal and retropharyngeal extension in $36 \%$ and $10 \%$ of patients, respectively. MR imaging had a high positive predictive value for both abscesses (0.98) and deep extension (0.86). Patients with large abscesses and widespread edema patterns had a more severe course of illness.

CONCLUSIONS: Emergency neck MR imaging can accurately describe the extent and nature of abscess formation in tonsillar infections.

ABBREVIATIONS: CRP = C-reactive protein; Gd = gadolinium; ITA = intratonsillar abscess; LOS = length of the hospital stay; PPV = positive predictive value; PTA = peritonsillar abscess; SLS = sublingual space; SMS = submandibular space; T2SI = T2-signal intensity; VS = visceral space

T onsillar infections are common, many are resolved conservatively, but complicated infections with abscesses still cause a substantial amount of morbidity and surgery. ${ }^{1}$ While common tonsillar infections can usually be treated on clinical grounds, emergency imaging may be required to identify abscess formation and rule out other diagnoses. ${ }^{2}$ The purpose of imaging is to differentiate abscesses from cellulitis and to describe the true extent of an abscess to select the appropriate treatment. Peritonsillar abscesses (PTAs) are collections of pus in the pharyngeal mucosal space between the tonsillar capsule and the pharyngeal constrictor muscle. While PTAs are not typically considered deep neck infections, they can

Received July 9, 2021; accepted after revision October 1.

From the Departments of Radiology (J. Heikkinen, J.N., T.H., M.N., K.M., J. Hirvonen) Otorhinolaryngology-Head and Neck Surgery (J.V., H.I.), and Oral and Maxillofacial Surgery (T.S.), University of Turku and Turku University Hospital, Turku, Finland.

Funding was provided by the University of Turku and the Sigrid Juselius Foundation.

Please address correspondence to Jussi Hirvonen, MD, PhD, Department of Radiology, Turku University Hospital, Kiinamyllynkatu 4-8, 20521 Turku, Finland; e-mail: jueshi@utu.fi; @jussihirvonen

- Indicates open access to non-subscribers at www.ajnr.org

Indicates article with online supplemental data.

http://dx.doi.org/10.3174/ajnr.A7368 become deeply extended abscesses if they rupture through the constrictor muscle to the deep neck spaces such as the parapharyngeal and retropharyngeal spaces.

Emergency neck imaging has traditionally been performed using contrast-enhanced CT. Previous literature on the diagnostic accuracy of CT has focused on deep neck infections, for which the positive predictive value (PPV) for abscesses is about $80 \%,{ }^{3-5}$ most likely due to the limited soft-tissue contrast of CT. In addition, previous literature on CT has suggested that some abscesses may be located within the tonsillar parenchyma and may not reach the peritonsillar space; these are called "intratonsillar abscesses" (ITAs). ${ }^{6}$ The diagnostic accuracy of the detection of ITA, PTA, and deep extension among patients undergoing emergency neck imaging is not well known, and the relative prevalence of these conditions also remains undetermined.

MR imaging provides superior soft-tissue contrast compared with $\mathrm{CT},{ }^{7,8}$ and emergency neck MR imaging is feasible even in acutely ill patients. ${ }^{9} \mathrm{MR}$ imaging can distinguish between reactive nonsuppurative edema and true abscesses in the deep neck spaces and has a high diagnostic accuracy in the detection of neck infections and abscesses. ${ }^{9}$ Therefore, MR imaging might be useful for illustrating the spectrum of tonsillar infections. To our knowledge, 
there are no systematic analyses of radiologic MR imaging findings in tonsillar infections and types of changes associated with a more severe course of illness.

This article aims to describe MR imaging findings regarding tonsillar infections and to investigate the significance of imaging findings related to clinical treatment and outcomes. To elucidate the full spectrum of tonsillar infections, we included patients with uncomplicated tonsillitis, PTA, and deeply extending abscesses.

\section{MATERIALS AND METHODS}

We obtained permission from the hospital district board for this retrospective cohort study in a single academic tertiary care referral center. Institutional review board review (approval or waiver) was not sought because it is not required by the national legislature for retrospective studies of existing data. The inclusion criteria were the following: 1) emergency MR imaging between April 1, 2013, and December 31, 2018, for suspected neck infection; 2) MR imaging evidence of infection: high signal of fat-suppressed T2-weighted Dixon images suggesting edema or high signal of fat-suppressed post-gadolinium (post-Gd) T1-weighted Dixon images suggesting abnormal tissue enhancement; 3) a final clinical diagnosis of a tonsillar infection; and 4) diagnostic image quality as determined by the radiologist reading the study. Cases were identified from the PACS and Radiology Information Systems using standard neck MR imaging codes, and data were cross-referenced with patients' medical files.

MR imaging was performed on an Ingenia 3T system using a dS HeadNeckSpine coil configuration (Philips Healthcare) and a Gd-based contrast agent (gadoterate meglumine, Dotarem; Guerbet). Details of the MR imaging protocol can be found in the Online Supplemental Data. The emergency radiology department of our institution has a dedicated emergency MR imaging service. At our institution, MR imaging has become the first-line imaging technique for most patients with suspected neck infections. For almost all patients, MR imaging was the only imaging method.

All imaging data were retrospectively reviewed by 2 fellowshiptrained neuroradiologists (J. Heikkinen and J. Hirvonen). To assess the diagnostic accuracy of the MR imaging findings, we used the following clinical reference standards: For infection, we used the final clinical diagnosis from the medical record, and for abscesses, we used the presence or absence of purulence or an abscess cavity during an operation if performed within 48 hours of MR imaging. The methods of surgical proof included open surgery, drainage, or puncture of pus. Because patients without abscesses are much less likely to undergo an operation than patients with abscesses (partial verification bias), we considered patients with no abscesses who recovered uneventfully following conservative treatment (including intravenous antibiotics) as representing true-negatives. The following data were extracted from medical records: age (years), sex (male/female), body mass index (kilogram/square meter), duration of symptoms before imaging (days), C-reactive protein (CRP, milligrams/liter), white blood cell count $\left(\times 10^{9} /\right.$ liter $)$, body temperature (degree Celsius), operation (yes/no), treatment in an intensive care unit (yes/no), and length of the hospital stay (LOS, days).

An abscess was defined as an abnormal nonenhancing T2hyperintense collection with a low ADC surrounded by abnormal tissue enhancement. We classified PTAs as being predominantly
Table 1: Patient characteristics ${ }^{\mathrm{a}}$

\begin{tabular}{lc}
\hline \multicolumn{1}{c}{ Characteristic } \\
\hline No. of patients & 132 \\
Age (mean) (yr) & $42(\mathrm{SD}, 19)$ \\
Male (No.) (\%) & $83(63 \%)$ \\
Female (No.) (\%) & $49(37 \%)$ \\
BMI (kg/m $\left.{ }^{2}\right)$ & 27.4 \\
CRP (mg/L) & $132^{\mathrm{a}}(\mathrm{SD}, 83)$ \\
WBC ( $\left.\times 10^{9} / \mathrm{L}\right)$ & $16^{\mathrm{b}}(\mathrm{SD}, 18)$ \\
Body temperature $\left({ }^{\circ} \mathrm{C}\right)$ & $37.6^{\mathrm{c}}(\mathrm{SD}, 0.8)$ \\
Duration of symptoms (days) & $5.6^{\mathrm{d}}(\mathrm{SD}, 5)$ \\
ICU (No.) (\%) & $17(13 \%)$ \\
LOS (mean) (days) & $3.9(\mathrm{SD}, 5.8)$ \\
Operation (No.) $(\%)$ & $104(79 \%)$ \\
\hline
\end{tabular}

Note:-BMI indicates body mass index; ICU, intensive care unit; WBC, white blood cell. ${ }^{a}$ Data available for a) 131, b) 131, c) 66, and d) 129 patients. Data are means or No. (\%).

superior, inferior, or widespread (both superior and inferior) relative to the craniocaudal midpoint of the palatine tonsil. Deep extension of an abscess was recorded as involvement of the parapharyngeal or retropharyngeal spaces. Uncomplicated PTA and deeply extending abscesses are collectively referred to as pharyngotonsillar abscesses. We also recorded whether abscesses had a multilocular morphology and whether the inflamed palatine tonsil had a striated appearance. We estimated the volume of the nonenhancing area of all the abscesses and measured the ADC and T2-signal intensity (T2SI) ratios between the abscess and the adjacent palatine tonsil. From fat-suppressed T2-weighted Dixon images, we evaluated edema as a high signal in the submandibular space (SMS, inferior to the mylohyoid muscle), sublingual space (SLS, superior to the mylohyoid muscle), and visceral space (VS, infrahyoid soft-tissue space including the larynx, surrounding strap muscles, and thyroid, also including edema in the anterior cervical space between the sternocleidomastoid muscle and the carotid space). To ensure the reliability of these consensus assessments, a third fellowship-trained neuroradiologist (M.N.) independently read a random sample of 60 patients for deep extension and edema patterns (SMS, SLS, VS). All radiologic assessments were conducted blinded to other medical and surgical information.

The results are expressed as percentages, means, and SDs. We used independent samples $t$-tests for comparing continuous variables and $\chi^{2}$ tests for comparing ordinal data. For assessing the diagnostic accuracy of detecting abscesses and deep extension, we formulated $2 \times 2$ tables (MR imaging versus surgical findings) and calculated the sensitivity, specificity, PPV, negative predictive value, and accuracy as previously described. ${ }^{9}$ We evaluated the interrater reliability of the assessments of the edema patterns using the Cohen $\kappa$. For the multivariate prediction model for deeply extending abscesses, we used a binary logistic regression model in which all statistically significant univariate predictors entered the model. All data were analyzed using SPSS Statistics for Mac (Version 26; IBM, 2019). $P$ values $<.05$ were considered statistically significant.

\section{RESULTS}

\section{Patient Population and Prior Procedures}

The study consisted of 132 patients (83 males, 49 females) with a clinically confirmed acute tonsillar infection (Table 1). One additional patient underwent MR imaging due to a suspected PTA but had to be excluded because of motion artifacts. The patients had a 

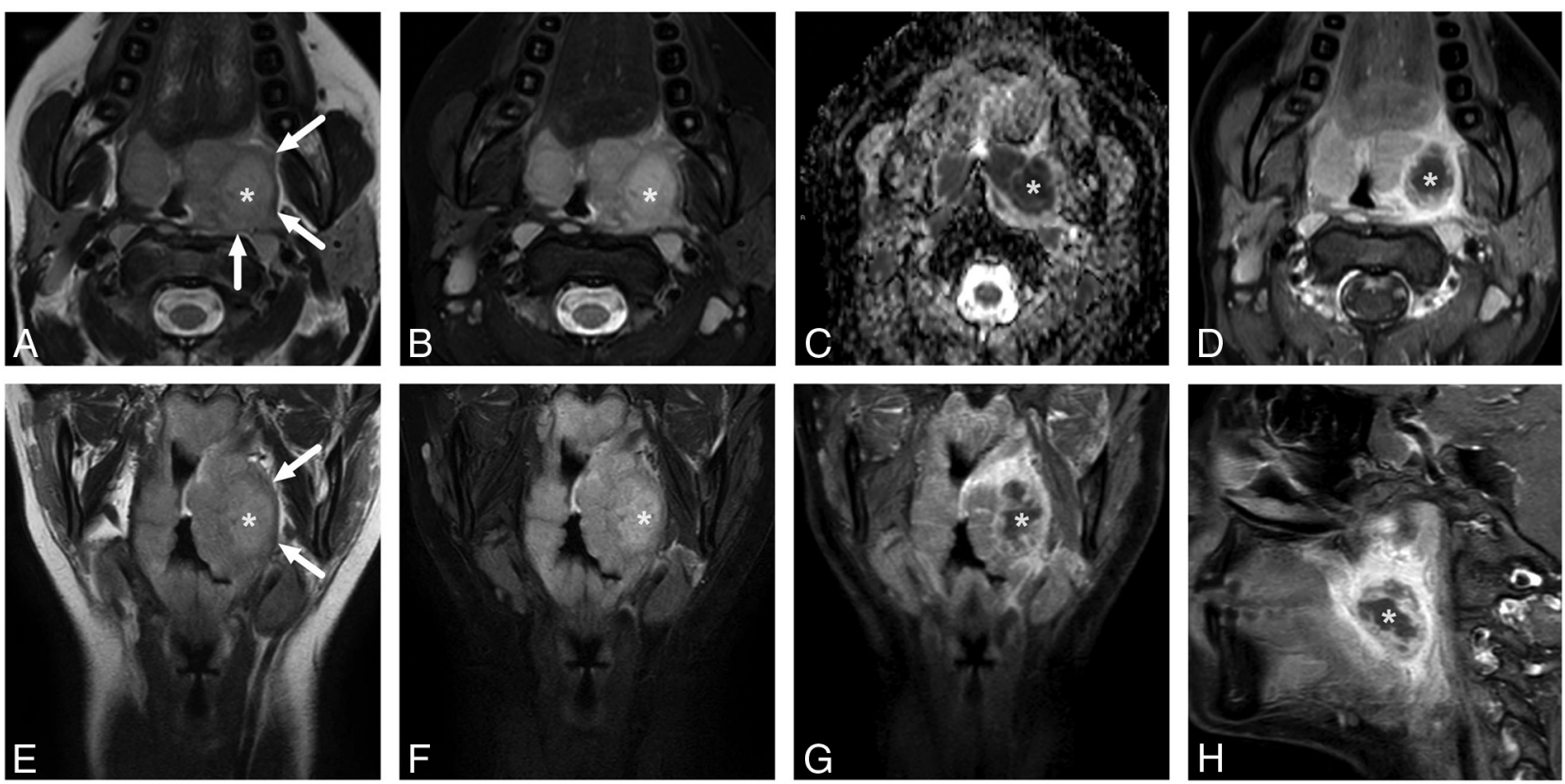

FIG 1. Uncomplicated PTA in an 18-year-old male patient with a sore throat. In-phase axial $(A)$ and coronal $(E)$ as well as fat-suppressed axial (B) and coronal (F) T2-weighted Dixon images reveal a slightly T2-hyperintense abscess (asterisk) lateral to the left palatine tonsil. The edematous pharyngeal constrictor muscle can be seen around the palatine tonsil (arrows in in-phase images $A$ and $E$ ). The abscess shows restricted diffusion on the ADC map (C) and a nonenhancing core surrounded by an enhancing rim in axial $(D)$, coronal $(G)$, and sagittal $(H)$ post-Gd fat-suppressed 7 -weighted Dixon images.

Table 2: Diagnostic accuracy of MR imaging in the detection of abscesses and deep extension (parapharyngeal, retropharyngeal involvement) in the abscesses ${ }^{\mathrm{a}}$

\begin{tabular}{lcc}
\hline \multirow{2}{*}{ MRI } & \multicolumn{2}{c}{ Operation } \\
\cline { 2 - 3 } \cline { 2 - 3 } Yes & No \\
\hline Yes & 96 & 2 \\
No & 2 & $19^{\mathrm{b}}$ \\
Deep extension & & \\
Yes & 36 & 5 \\
No & 6 & 57 \\
\hline
\end{tabular}

a Sensitivity, 0.98; specificity 0.90; PPV, 0.98; negative predictive value, 0.90; accuracy, 0.97. In the complications, sensitivity, 0.88; specificity, 0.90; PPV, 0.86; negative predictive value, 0.92 ; accuracy 0.89 .

${ }^{\mathrm{b}}$ Conditions of 4 patients were surgically confirmed, and the diagnoses of 15 patients were based on clinical assessment.

mean age of 42 years (range, 9-82 years). The mean duration of symptoms before imaging was about 5 days. One hundred four (79\%) patients underwent an operation, and 17 (13\%) patients required treatment in the intensive care unit. The mean LOS was 4 days (range, $0-55$ days). Imaging showed evidence of $\geq 1$ abscess (101 unilateral, 11 bilateral) in 112 patients (85\%) (Fig 1) and of parapharyngeal and retropharyngeal extension in 40 (36\%) and 11 (10\%) patients, respectively.

Among the patients who required imaging, 88 (68\%) had not undergone any prior procedure and were imaged swiftly because of suspected deep abscess, whereas 42 (32\%) patients had undergone a previous procedure (needle aspiration and/or incision and drainage). Most of these prior procedures (84\%) had not yielded pus. Nevertheless, these patients were still suspected of having an abscess on clinical grounds, and imaging showed an abscess in $86 \%$ of them.

\section{Diagnostic Accuracy for Abscesses and Deep Extension}

All patients had a final clinical diagnosis of tonsillar or pharyngeal infection. Of those with an abscess, 98 (89\%) underwent an operation and an abscess was surgically confirmed in 96 patients, suggesting a PPV of 0.98 . We had 2 patients with false-positive findings in whom no purulence was found. Six patients underwent an operation without MR imaging evidence of abscess; among these, 4 had true-negative findings and 2 had false-negative findings. Assuming 15 more patients with true-negative findings (ie, patients with a tonsillar infection but no abscess who recovered uneventfully following conservative treatment), we found a sensitivity, specificity, and accuracy of $0.98,0.90$, and 0.97 , respectively, for an MR imaging diagnosis of an abscess (Table 2). In our experience, craniocaudal or lateral extension of an abscess far from the tonsil is the most reliable imaging sign of parapharyngeal or retropharyngeal extension (Fig 2). This morphologic assessment of deep extension had an overall sensitivity, specificity, and accuracy of $0.88,0.90$, and 0.89 , respectively, as well as substantial agreement in the interobserver analysis (92\% agreement, $\kappa=0.75$ ).

\section{Imaging Characteristics of Abscesses (Only True- Positives)}

In total, we found 110 abscesses by MR imaging (2 false-positive findings were excluded) (Table 3 ). The mean maximal abscess diameter was $33 \mathrm{~mm}$, and volume was $3.2 \mathrm{~mL}$. Most (ie, 69, 63\%) of the abscesses were multilocular, and 78 (71\%) had a striated tonsillar appearance in post-Gd T1-weighted images. The mean ADC value was 0.52 (compared with 0.75 in tonsillar tissue; ADC ratio, 0.72 ). The average T2SI ratio between the abscess and the tonsil was 1.3 (Fig 3). Among the patients with an abscess, only 

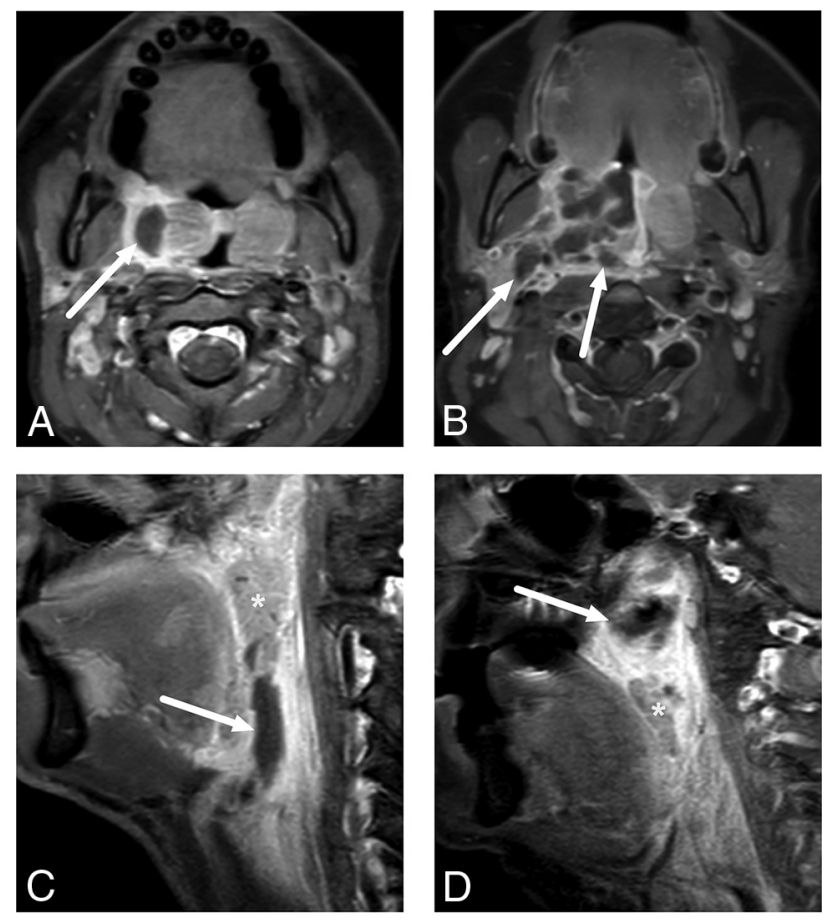

FIG 2. Examples of uncomplicated PTA $(A)$ and abscesses with deep extension $(B-D)$ in different patients. In uncomplicated PTA, the abscess is retained within the pharyngeal constrictor muscle $(A)$. In axial images, a deeply extending PTA is often lobulated and reaches laterally toward the parapharyngeal space, beyond the pharyngeal constrictor muscle $(B)$. In sagittal images, a deeply extending PTA can reach far inferiorly $(C)$ or superiorly $(D)$ from the palatine tonsil (asterisk). The arrows denote abscesses.

Table 3: Imaging characteristics ${ }^{a}$

\begin{tabular}{lc}
\hline Outcome & \\
\hline Abscess characteristics & $110(83 \%)^{\mathrm{b}}$ \\
Maximal abscess diameter (mm) & $33(\mathrm{SD}, 22)$ \\
Volume (mL) & $3.2(\mathrm{SD}, 4.4)$ \\
Multilocular & $69(63 \%)$ \\
Striated & $78(71 \%)$ \\
T2SI ratio & $1.3(\mathrm{SD}, 0.3)$ \\
ADC & $0.52(\mathrm{SD}, 0.15)$ \\
Abscess location & \\
Superior & $47(43 \%)$ \\
Inferior & $37(34 \%)$ \\
Widespread & $26(24 \%)$ \\
Edema patterns & \\
SMS & $121(92 \%)$ \\
SLS & $31(24 \%)$ \\
VS & $95(72 \%)$ \\
Deep extension & $41(37 \%)$ \\
Parapharyngeal & $40(36 \%)$ \\
Retropharyngeal & $11(10 \%)$ \\
Both & $10(9 \%)$ \\
\hline a Values are No. (\%) or means. & \\
b True-positives. &
\end{tabular}

$8 \%$ had a higher ADC and $11 \%$ had a lower T2SI in the abscess than in the tonsil.

Of the abscesses, $43 \%$ were considered superior; $34 \%$, inferior; and $24 \%$, widespread (with superior and inferior extensions) relative to the palatine tonsil. Superior-type abscesses were more

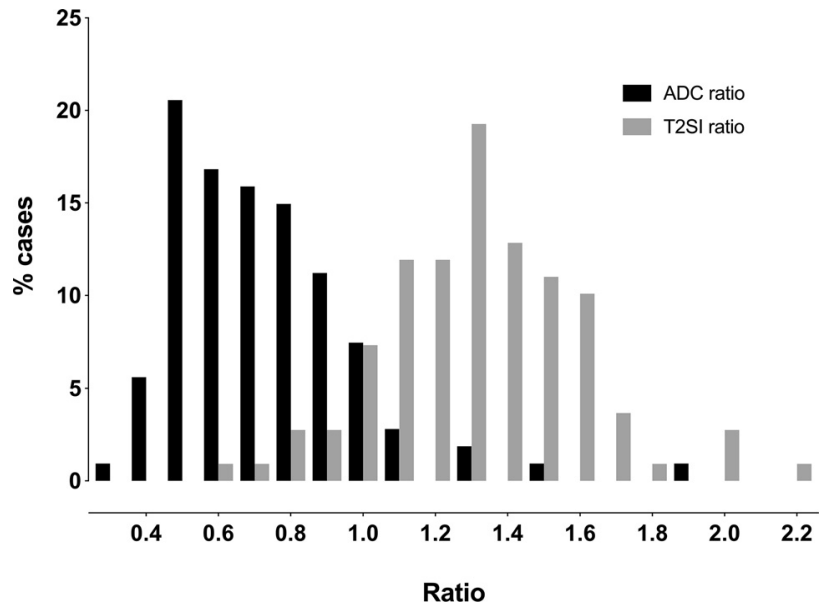

FIG 3. Frequency distributions of $A D C$ and $T 2 S I$ ratios between the abscesses and the adjacent palatine tonsils. In most patients, the abscesses had a lower ADC and higher T2SI than the palatine tonsils.

common among the younger patients (mean age, 37 years versus 44 and 47 years for inferior and widespread types, $P=.046)$ and among women (53\% of all 3 abscess types compared with $35 \%$ among men, $P=.001)$. The superior abscesses were also smaller (20 versus $30 \mathrm{~mL}$ and $55 \mathrm{~mL}, P=.004$ ), had fewer deep extensions (22\% versus $49 \%$ and $29 \%, P=.003$ ), and the associated LOS was shorter (2.8 days versus 3.1 and 5.6 days, $P=.006$ ) compared with inferior and widespread types, respectively. Larger widespread abscesses had the most admissions to the intensive care unit (50\% of all intensive care unit admissions, $P=.045$ ) and the longest mean LOS (5.6 days versus 2.8 and 3.1 days, $P=.006$ ).

\section{Edema Patterns}

SMS edema was the most common (92\%), followed by VS (72\%) and SLS (24\%) edema (Fig 4). The SMS and SLS edema patterns were not significantly differently distributed among the abscess locations, whereas VS edemas were most common in inferiorly located abscesses ( $42 \%$ versus $33 \%$ and $25 \%$ in superior and widespread types, $P=.001)$. The patients with VS edemas had larger abscesses ( 3.8 versus $1.5 \mathrm{~mL}, P<.001$ ) and higher CRP (142 versus $90, P=.005)$ than those without. Similarly, the patients with SLS edemas had larger abscesses (4.6 versus $2.7 \mathrm{~mL}, P=.047$ ). In the interobserver analysis, these edema patterns had moderateto-substantial agreement $(93 \%, 85 \%$, and $78 \%$ agreement, and $\kappa=0.47,0.64$, and 0.52 for SMS, VS, and SLS, respectively).

\section{Prediction of Parapharyngeal and Retropharyngeal Deep Extension Using Imaging Findings}

The patients with deeply extended diseases who underwent an operation were older (47 versus 39 years, $P=.024$ ) and had higher CRP values (173 versus $116, P<.001$ ), larger abscesses (5.4 versus $1.9 \mathrm{~mL}, P<.001$ ), a higher prevalence of VS (95\% versus $62 \%, P<.001$ ), SLS ( $42 \%$ versus $15 \%, P<.001$ ), and inferior locations of abscesses (49\% versus $22 \%$ and $29 \%$ in the superior and widespread types, $P=.003$ ) than the patients with uncomplicated PTAs (Table 4).

In the multivariate analysis, an extended disease was predicted by abscess volume $(P=.012)$ CRP $(P=.017)$, and SLS edema 

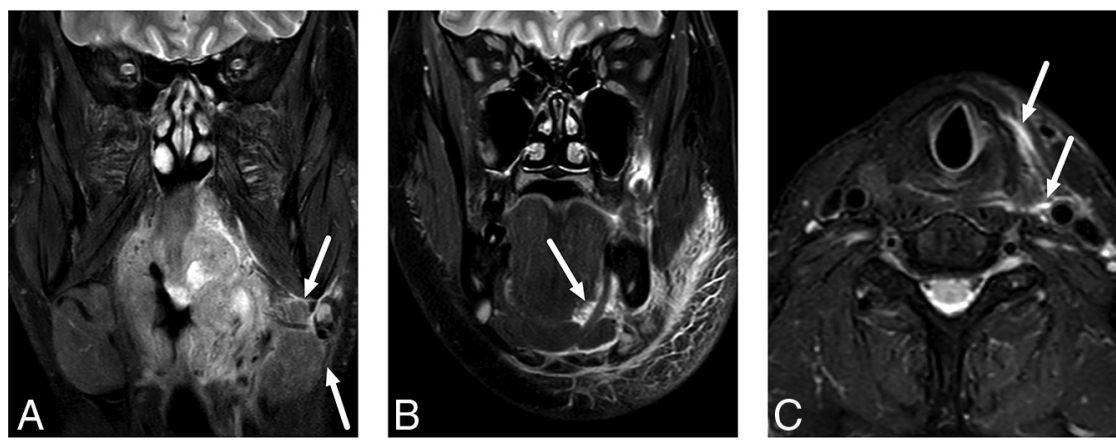

FIG 4. Examples of SMS (A), SLS (B), and VS (C) edema patterns on fat-suppressed T2-weighted Dixon images. Arrows indicate edema.

Table 4: Univariate and multivariate modeling of deep extension of abscesses

\begin{tabular}{lcrrcc}
\hline & \multicolumn{2}{c}{ Univariate } & & \multicolumn{2}{c}{ Multivariate } \\
\cline { 2 - 3 } \cline { 5 - 6 } & Statistic $^{\mathbf{a}}$ & $\boldsymbol{P}$ Value & & Odds Ratio & $\boldsymbol{P}$ Value \\
\hline Age & -2.28 & .024 & & 1.03 & .054 \\
CRP & -3.81 & $<.001$ & & 1.01 & .017 \\
WBC & -0.284 & .777 & & \\
Location & 11.9 & .003 & & 1.31 & .414 \\
Abscess volume & -4.50 & $<.001$ & & 1.00 & .012 \\
SMS & 0.993 & .501 & & \\
SLS & 11.4 & .001 & 3.43 & .032 \\
VS & 15.8 & $<.001$ & 3.09 & .173 \\
\hline
\end{tabular}

Note:-WBC indicates white blood cell count.

${ }^{a} T$-value for continuous variables, $\chi^{2}$ for nominal variables.

$(P=.032)$ in patients with a surgically confirmed pharyngotonsillar abscess. This model correctly classified $77 \%$ of the patients and explained $47 \%$ of the variance (Nagelkerke $\mathrm{R}^{2}$ ) (Table 4 ).

\section{DISCUSSION}

This study advances our knowledge of the spectrum of tonsillar infections in several ways. First, MR imaging has a high diagnostic accuracy in detecting abscesses and parapharyngeal and retropharyngeal deep extension. Second, the T2SI of peritonsillar abscesses can be visually close to that of tonsillar tissue and not easily distinguishable from the latter. In addition, the ADC between the abscess and tonsil is often visually similar. These observations support the routine use of Gd-based contrast agents. Third, we found no evidence of intratonsillar abscesses because, by definition, none of the cases had tonsillar tissue surrounding the abscess on all sides; that is, all the abscesses reached the superior constrictor muscle and were, thus, at least peritonsillar in nature. Finally, the patients with large abscesses and widespread edema patterns seemed to have more severe courses of illness.

The primary objective of emergency imaging is to provide a correct diagnosis and an anatomic basis for managing deep neck infections. Although PTAs do not typically require imaging, the patients in the current study underwent emergency MR imaging because either prior procedures had not yielded pus or a deep extension of the disease was primarily suspected. In our study of 98 patients with a radiologically diagnosed abscess who were later treated surgically, 96 had an abscess verified by the criterion standard. Of the false-positives, 1 patient had a hematoma after many puncture attempts. The second case was a very small abscess identified on MR imaging, and no purulence was identified during the operation. We found a sensitivity, specificity, and accuracy of $0.98,0.90$, and 0.97 , respectively, for an MR imaging diagnosis of a pharyngotonsillar abscess. These results compare well with those of previous CT studies with the sensitivity, specificity, and accuracy of $<0.90$. $4,10,11$ Among the patients needing imaging for tonsillar infection, 37\% had a deep extension to the parapharyngeal or retropharyngeal spaces. For the detection of parapharyngeal or retropharyngeal extension, the sensitivity, specificity, and accuracy were $0.88,0.90$, and 0.89 , respectively, and interobserver agreement was substantial. In our experience, parapharyngeal or retropharyngeal extension should be suspected if the abscess reaches outside the palatine tonsil borders either laterally, superiorly, or inferiorly (Fig 2). Surgical literature shows that most cases of parapharyngeal abscesses are associated with concomitant PTA, suggesting that they are often direct extensions of PTA. ${ }^{12}$ Our current results indicate that deep extension can be detected or ruled out accurately by MR imaging.

An ITA is thought to be a purulent collection completely within the tonsillar parenchyma, often considered trapped pus inside a tonsillar crypt. It is suggested to carry a more favorable prognosis and could be treated with less invasive methods than PTA. ${ }^{13-17}$ We rigorously scrutinized all the abscesses for ITAs but could not find a single case in which the abscess was completely surrounded by the tonsillar parenchyma. ITA versus PTA is likely a nomenclature issue, and these 2 types of abscesses may reflect different stages on a spectrum of the same disease, with a variable amount of pus inside the tonsillar parenchyma. It is unknown to what extent CT can differentiate a swollen pharyngeal constrictor muscle and tonsillar parenchyma, therefore between ITAs and PTAs, whereas MR imaging can differentiate them by virtue of ADC maps.

Regarding the location of abscesses, superior types were slightly more common than inferior and widespread types and tended to be smaller and less severe cases overall, with the shortest LOS. Bilateral abscesses were diagnosed in $10 \%$ of all patients with abscesses. On average, concomitant contralateral PTAs are discovered intraoperatively in $5.5 \%$ of patients. ${ }^{18}$ The rate of bilateral PTAs found using CT is not known but may be lower than that found using MR imaging.

Most abscesses have lower ADC values and higher T2SI than the tonsillar tissue itself. In some cases, abscess fluid can be so purulent that the T2SI is not very high (Fig 1). On the other hand, normal tonsillar tissue has a low ADC by virtue of high cellularity, so differentiating the abscess from the tonsil may be challenging. Although only $8 \%$ of the patients had higher ADCs and $11 \%$ of the patients had lower T2SI in the abscess than in the tonsil, in our experience, post-Gd T1-weighted images are required to delineate pharyngotonsillar abscesses accurately. Future studies should measure the diagnostic accuracy and interobserver agreement when 
using only DWI and T2-weighted imaging without a Gd-based contrast agent in cases of pharyngotonsillar abscesses.

Almost all patients with a tonsillar infection had SMS edema, reflecting the anatomic free communication with these spaces. Whether the edema spreads directly or via lymphatic spread is unclear. Tonsillar infections that produce widespread edema patterns are likely to be more severe because the edema crosses the boundaries between the soft-tissue compartments. This finding was demonstrated in our study because the patients with VS and SLS edemas had larger abscesses, and the patients with VS edemas also had significantly higher CRP and longer LOS. In the multivariate analysis, an extended abscess (parapharyngeal or retropharyngeal) was also predicted by SLS edema with a surgically confirmed pharyngotonsillar abscess, even when controlling for CRP. Conversely, an operation rarely revealed an extended abscess in patients without VS and SLS edemas. This finding was especially prominent among patients without VS edemas because only 2 patients had an extended abscess without it.

The strengths of the current study include a large sample size, high-quality $3 \mathrm{~T}$ MR imaging with a Gd-based contrast agent and DWI, systematic neuroradiologic evaluation of MR imaging findings, thorough clinical characterization, and surgical confirmation of abscesses. However, limitations need to be addressed. A major limitation of our study is that we did not directly compare MR imaging findings in the same patients with those from CT, an established imaging method in current clinical practice. Therefore, we cannot make claims about the overall superiority of MR imaging over CT in patients with tonsillar infections. MR imaging may not always be suitable or available for all patients, and these results may not apply to all facilities. Very few direct comparisons between MR imaging and CT have been made, and future prospective comparative trials should be conducted. This question could also be explored with the increased soft-tissue characterization of dual-energy CT. ${ }^{19}$ A recent study showed that a lower tube voltage of $80 \mathrm{kV}$ (peak) improved the delineation of PTA in comparison with the typical $120-\mathrm{kVp}$ tube voltage. ${ }^{20}$

Furthermore, our study was retrospective in nature; therefore, medical and surgical records may have been incomplete or imprecise. Indications for imaging may have varied; thus, the current results may be biased. Because of partial verification bias (patients without abscesses are much less likely to undergo an operation than patients with abscesses), the proportions of true- and falsenegatives are not reliable. Unverified false-negatives (small undetected abscesses that do not require an operation) may overestimate the sensitivity and underestimate the specificity.

Our study may have selection bias toward more severely ill patients, which could have limited our ability to detect ITAs. The surgical results were also sometimes unclear, though in very few cases. The interpretation of some of the MR imaging findings may be subjective: We found moderate-to-substantial interobserver agreement for the edema patterns. Finally, this study is limited by the lack of a control group, and the findings only pertain to patients undergoing emergency MR imaging.

\section{CONCLUSIONS}

Emergency neck MR imaging can accurately describe the extent and nature of abscess formation in tonsillar infections.
Disclosure forms provided by the authors are available with the full text and PDF of this article at www.ajnr.org.

\section{REFERENCES}

1. Bochner RE, Gangar M, Belamarich PF. A clinical approach to tonsillitis, tonsillar hypertrophy, and peritonsillar and retropharyngeal abscesses. Pediatr Rev 2017;38:81-92 CrossRef Medline

2. Steyer TE. Peritonsillar abscess: diagnosis and treatment. Am Fam Physician 2002;65:93-96 Medline

3. Elden LM, Grundfast KM, Vezina G. Accuracy and usefulness of radiographic assessment of cervical neck infections in children. $J$ Otolaryngol 2001;30:82-89 CrossRef Medline

4. Vural C, Gungor A, Comerci S. Accuracy of computerized tomography in deep neck infections in the pediatric population. Am J Otolaryngol 2003;24:143-48 CrossRef Medline

5. Freling N, Roele E, Schaefer-Prokop C, et al. Prediction of deep neck abscesses by contrast-enhanced computerized tomography in $\mathbf{7 6}$ clinically suspect consecutive patients: deep neck abscesses predicted by CECT. Laryngoscope 2009;119:1745-52 CrossRef Medline

6. Blair AB, Booth R, Baugh R. A unifying theory of tonsillitis, intratonsillar abscess and peritonsillar abscess. Am J Otolaryngol 2015;36:51720 CrossRef Medline

7. Wang B, Gao BL, Xu GP, et al. Images of deep neck space infection and the clinical significance. Acta Radiol 2014;55:945-51 CrossRef Medline

8. Muñoz A, Castillo M, Melchor MA, et al. Acute neck infections: prospective comparison between CT and MRI in 47 patients. J Comput Assist Tomogr 2001;25:733-41 CrossRef Medline

9. Nurminen J, Velhonoja J, Heikkinen J, et al. Emergency neck MRI: feasibility and diagnostic accuracy in cases of neck infection. Acta Radiol 2021;62:735-42 CrossRef Medline

10. Stone ME, Walner DL, Koch BL, et al. Correlation between computed tomography and surgical findings in retropharyngeal inflammatory processes in children. Int J Pediatr Otorhinolaryngol 1999;49:121-25 CrossRef Medline

11. Page NC, Bauer EM, Lieu JE. Clinical features and treatment of retropharyngeal abscess in children. Otolaryngol Head Neck Surg 2008;138:300-06 CrossRef Medline

12. Klug TE, Fischer AS, Antonsen C, et al. Parapharyngeal abscess is frequently associated with concomitant peritonsillar abscess. Eur Arch Otorhinolaryngol 2014;271:1701-07 CrossRef Medline

13. Wang AS, Stater BJ, Kacker A. Intratonsillar abscess: 3 case reports and a review of the literature. Int $J$ Pediatr Otorhinolaryngol 2013;77:605-07 CrossRef Medline

14. Singh GB, Kumar D, Arora R, et al. A rare case of intratonsillar abscess in an adult. Clin Pract 2015;5:804 CrossRef Medline

15. Ahmed Ali S, Kovatch KJ, Smith J, et al. Predictors of intratonsillar abscess versus peritonsillar abscess in the pediatric patient. Int $J$ Pediatr Otorhinolaryngol 2018;114:143-46 CrossRef Medline

16. Ahmed Ali S, Kovatch KJ, Smith J, et al. Predictors of intratonsillar versus peritonsillar abscess: a case-control series. Laryngoscope 2019;129:1354-59 CrossRef Medline

17. Giurintano JP, Kortebein S, Sebelik M, et al. Intratonsillar abscess: a not-so-rare clinical entity. Int J Pediatr Otorhinolaryngol 2019;119:3840 CrossRef Medline

18. Klug TE, Rusan M, Fuursted K, et al. Peritonsillar abscess: complication of acute tonsillitis or Weber's glands infection? Otolaryngol Head Neck Surg 2016;155:199-207 CrossRef Medline

19. May MS, Wiesmueller M, Heiss R, et al. Comparison of dual- and single-source dual-energy CT in head and neck imaging. Eur Radiol 2019;29:4207-14 CrossRef Medline

20. Scholtz JE, Hüsers K, Kaup M, et al. Evaluation of image quality and dose reduction of $80 \mathrm{kVp}$ neck computed tomography in patients with suspected peritonsillar abscess. Clinical Radiol 2015;70:e67-73 CrossRef Medline 